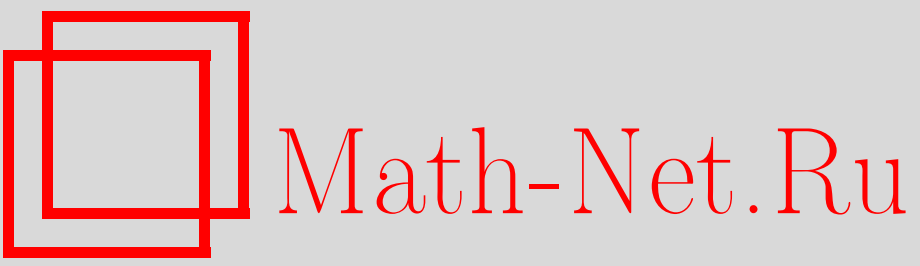

М. В. Мещеряков, Оценки секционных кривизн римановых симметрических пространств, УМН, 1996, том 51, выпуск 1, 157-158

DOI: https://doi.org/10.4213/rm931

Использование Общероссийского математического портала Math-Net.Ru подразумевает, что вы прочитали и согласны с пользовательским соглашением

http://www . mathnet.ru/rus/agreement

Параметры загрузки:

IP : 52.90 .164 .192

26 апреля 2023 г., 16:27:08 


\title{
ОЦЕНКИ СЕКЦИОННЫХ КРИВИЗН РИМАНОВЫХ СИММЕТРИЧЕСКИХ ПРОСТРАНСТВ
}

\author{
М.В. МЕШЕРЯков
}

Римановы симметрические пространства - хорошо изученный класс римановых многообразий. Тем не менее в литературе, видимо, отсутствует исследование вопроса об оценках их секционных кривизн, кроме случая симметрических пространств ранга 1 , изучавшегося в связи с проблемой зашемленности кривизны (см. [1], [2]). Нашей целью будет вычисление критических значений секционной кривизны биинвариантных римановых метрик на некоммутативных компактных группах Ли и получение оценок секционной кривизны римановых симметрических пространств ранга, большего 1. Достаточно ограничиться случаем компактных пространств, так как при переходе к двойственному пространству меняется только знак кривизны. Напомним, что компактные симметрические пространства характеризуются неотрицательностью кривизны. Нулевое значение кривизны всегда достигается, если ранг пространства больше 1.

Пусть $\mathscr{G}$ - алгебра Ли компактной группы Ли $G$, снабженной биинвариантной римановой метрикой $\langle\cdot, \cdot\rangle$. Секционная кривизна $K_{\sigma}$ этой метрики вычисляется по классической формуле $4 K_{\sigma}(x, y)=\langle[x, y],[x, y]\rangle$. Здесь $x, y \in \mathscr{G}$ образуют ортонормированный базис двумерной плоскости $\sigma$, и $[x, y]$ - коммутатор в алгебре Ли $\mathscr{G}$ (см. [3]). Пусть $G_{2}(\mathscr{G})$ - грассманиан двумерных плоскостей в $\mathscr{G}$ и $S=\{(x, y):\langle x, x\rangle=\langle y, y\rangle=1,\langle x, y\rangle=0\}$ - соответствующее многообразие Штифеля. Определим критические точки функции $K_{\sigma}: S \rightarrow \mathbb{R}$. Непосредственным вычислением доказывается

ПреДЛОЖениЕ 1. Точка $(x, y) \in S$ является критической точкой функции $K_{\sigma}$ тогда и только тогда, когда выполнены следующие условия критичности в алгебре $\mathscr{G}$ :

$$
[x, y]=z, \quad[y, z]=4 K_{\sigma}(x, y) x, \quad[z, x]=4 K_{\sigma}(x, y) y .
$$

При $K_{\sigma}(x, y)>0$ условия критичности означают, что $x, y$ и $z$ образуют базис трехмерной неабелевой подалгебры компактной алгебры Ли $\mathscr{G}$. Отсюда вытекает описание критических точек функции $K_{\sigma}: G_{2}(\mathscr{G}) \rightarrow \mathbb{R}$.

Теорема 1. Отличные от нуля критические значения функции секционной кривизны $K_{\sigma}$ бичнвариантной римановой метрики на абелевой компактной әруппе Ли достигаются на двумерных плоскостях $\sigma$, допускающих вложсене в простую трехмерную подалгебру алгебры Ли $\mathscr{G}$.

Трехмерные простые подгруппы группы $G$ обладают важными геометрическими свойствами.

ПрЕДЛОжЕнИЕ 2. Простье группь ранга 1 в компактной неабелевой группе Ли являются вполне геодезическими подмногообразиями постоянной положительной кривизньц.

Теорема 2. Отличные от нуля критические значения секчионной кривизны биинвариантной римановой метрики на неабелевой компактной группе Ли совпадают $c$ кривизнами ее простых подгрупп ранга 1.

Вычисление указанных в теореме 2 кривизн сводится к вычислению кривизны формы Киллинга алгебры Ли $\mathrm{su}(2)$ и определению нормировочных коэффициентов $\delta$, связьвающих форму Киллинга алгебры $\mathrm{su}(2)$ с ограничением $\langle\cdot, \cdot\rangle$ на трехмерную простую подалгебру. Коэффициент $\delta$ зависит тем самым от вложения $\mathrm{su}(2)$ в $\mathscr{G}$.

ПреДЛОЖЕнИЕ 3. Пусть $\varphi: \operatorname{su}(2) \rightarrow \mathscr{G}-$ вложение в $\mathscr{G} u\langle\varphi(x), \varphi(y)\rangle=\delta(-b(x, y))$, где $b$ - форма Киллинга алгебры $\mathrm{su}(2)$. Тогда секционная кривизна соответствующей подгруппы равна $1 / 8 \delta$.

Кривизна формы Киллинга алгебры $\mathrm{su}(2)$ равна $1 / 8$. При умножении метрики на $\delta$ секционная кривизна умножается на $1 / \delta$. Итак, $1 / 8 \delta$ - искомая кривизна.

Ограничимся в дальнейшем случаем полупростых алгебр Ли, где вычисление $\delta$ опирается на теорию представлений алгебры $\mathrm{su}(2)$. Выбор метрики $\langle\cdot, \cdot \cdot\rangle$ на $\mathscr{G}$ подчиним условию нормировки: длина старшего вектора системы корней алгебры $\mathscr{G}$ равна $\sqrt{2}$. Вместо формы Киллинга $b(x, y)=4 \operatorname{Tr} x y$ на $\mathrm{su}(2)$ возьмем форму $b_{0}(x, y)=-\operatorname{Tr} x y$. Форма $b_{0}$ удовлетворяет условию нормировки, и ее кривизна равна $1 / 2$. В этих предположениях число $\delta$ будет натуральным 
числом, называемым индексом Дынкина подалгебры (см. [5]). Аналогично определяется индекс $i(\rho)$ линейного представления $\rho$ простой алгебры Ли. Индекс $j \varphi$ вложения $\varphi: \operatorname{su}(2) \rightarrow \mathscr{G}$, coгласно [5], равен отношению индекса $i(a d \mid \mathrm{su}(2))$ ограничения присоединенного представления $a d$ алгебры $\mathscr{G}$ к индексу самого $a d$. Разлагая $a d \mid \operatorname{su}(2)$ в сумму неприводимых представлений $\rho_{1}, \ldots, \rho_{m}$, получаем $j_{\varphi}=\sum_{s=1}^{m} i\left(\rho_{s}\right) / i(a d)$. Но $i\left(\rho_{s}\right)=1 / 6 n_{s}\left(n_{s}+1\right)\left(n_{s}+2\right)$, где $n_{s}-$ числовая отметка на схеме Дынкина алгебры su(2), задающая представление $\rho_{s}$ (см. [5]). Отсюда приходим к формуле для секционной кривизны подалгебры $\varphi(\mathrm{su}(2))$ :

$$
K_{\sigma}=3 i(a d) / \sum_{s=1}^{m} n_{s}\left(n_{s}+1\right)\left(n_{s}+2\right) .
$$

В полупростой алгебре Ли всегда имеются подалгебры индекса 1. Поэтому максимумы секционной кривизны реализуются на таких подалгебрах. Более того, сравнение этого факта с классификацией глобально минимальных поверхностей реализующего типа в односвязных компактных группах Ли, найденной А. Т. Фоменко (см. [1]), приводит к следующему утверждению.

Теорема 3. Глобально минимальнье поверхности реализующего типа в компактной односвязной группе Ли имеют кривизну, равную максимуму секционных кривизн биинвариантной метрики.

Указанный в теореме 3 максимум, как и другие критические значения $K_{\sigma}$, дает

Теорема 4. Отличные от нуля критические значения секционной кривизны метрики Киллинга полупростой компактной группь Ли вычисляются по формуле

$$
K_{\sigma}=3 / \sum_{s=1}^{m} n_{s}\left(n_{s}+1\right)\left(n_{s}+2\right) .
$$

Максимальное значение $K_{\sigma}$ равно $1 / 2 i(a d)$.

Индекс присоединенного представления простой компактной алгебры Ли можно вычислить, зная отношение длин корней, пользуясь некоторыми соображениями из [6] (см. §4, гл. I).

ПредЛОЖенИЕ 4. Значения $i(a d)$ простых алгебр Ли даются следующим списком: $A_{n}(2(n+1)), B_{n}(2(2 n-1)), C_{n}(4(n+1)), D_{n}(4(n-1)), G_{2}(8), F_{4}(18), E_{6}(24), E_{7}(36)$, $E_{8}(60)$.

В скобках указаны значения индекса $i(a d)$.

Поскольку симметрические пространства вкладываются как вполне геодезические подмногообразия в их группы изометрий (картановская модель), то из теоремы 4 вытекает

Теорема 5. Секционная кривизна неприводимого компактного (соответственно, некомпактного) симметрического пространства не больие (соответственно, не меньше) чем $\pm 1 / i(a d)$ при условии, что метрика пространства индуцируется формой Киллинга.

Полученные оценки кривизны позволяют выписать явные оценки значений объемов глобально минимальных поверхностей в симметрических пространствах (см. приложение в [7]).

\section{СПИСОК ЛИТЕРАТУРЫ}

[1] Фоменко А. Т. Вариационные методы в топологии. М.: Наука, 1982. [2] Валиев Ф. М. // Сиб. матем. журн. 1979. Т. 20. № 2. С. 248-262. [3] Милнор Дж. Теория Морса. М.: Мир, 1965. [4] Кобаяси Ш., Номидзу К. Основы дифференциальной геометрии. Т. 1, 2. М.: Наука, 1981. [5] Винберг Э. Б., Горбацевич В. В., Онищик А. Л. // Итоги науки и техники. Соврем. пробл. матем. Фундам. напр. Т. 41. М.: ВИНИТИ, 1989. С. 5-258. [6] Спрингер Т. А., Штейнберг Р. // Семинар по алгебраическим группам. М.: Мир, 1973. С. 162-262. [7] Дао Чонг Тхи, Фоменко А. Т. Минимальные поверхности и проблема Плато. М.: Наука, 1987. 